\title{
Modelagem de Equações Estruturais Aplicada no Estudo de Causalidade entre Maturidade e Processos Organizacionais
}

\section{Structural Equation Modeling Applied to the Study of Causality between Maturity and Organizational Processes}

\author{
Adriano Rodrigues de Melo \\ Universidade Federal do Paraná - UFPR, Curitiba, PR \\ melo.a.rodrigues@gmail.com \\ Vanessa Ferreira Sehaber \\ Universidade Federal do Paraná - UFPR, Curitiba, PR \\ vsehaber@gmail.com \\ Jair Mendes Marques \\ Universidade Federal do Paraná - UFPR, Curitiba, PR \\ jair.marques@utp.br \\ Alfredo Iarozinski Neto \\ Universidade Tecnológica Federal do Paraná - UTFPR, Curitiba, PR \\ alfredo.iarozinski@gmail.com
}

Resumo: Este trabalho apresenta um estudo das relações entre construtos associados à maturidade organizacional e níveis de efetividade de processos empresariais. Busca quantificar e identificar os processos/construtos que melhor predizem o fator maturidade organizacional. Os métodos utilizados para determinar essa relação foram a análise fatorial exploratória e a modelagem de equações estruturais. Os dados utilizados consistem de uma planilha com 299 observações por 42 variáveis. Por meio dos resultados obtidos, verificou-se que todos os processos considerados são bons preditores quando avaliados em relações individuais e diretas, sendo o processo Desenvolvimento de Visão e Estratégia o mais significante, seguido dos processos

Recebido em 15/04/2013 - Aceito em 20/05/2013.

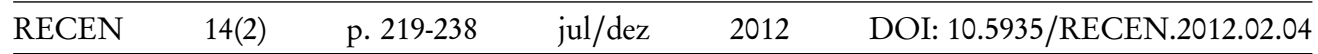


Revista Ciências Exatas e Naturais, Vol.14, n², Jul/Dez 2012

Gerência do Atendimento ao Consumidor e Marketing de Produtos e Serviços.

Palavras-chave: causalidade; maturidade; modelagem de equações estruturais; processos.

Abstract: This work is a study of the relationship between constructs related to organizational maturity and levels of effectiveness in business processes. It seeks to quantify and identify the processes/constructs that best predict organizational maturity factor. The methods used to determine this relationship were the exploratory factor analysis and the structural equation modeling. The data used in this work consists of a spreadsheet with 299 observations for 42 variables. Through the obtained results, it was found that all processes are considered good predictors when evaluated on individual and direct relationship, however the process Development of Vision and Strategy is seen as the most significant, followed by the processes Management's Customer Service and Marketing of Products and Services.

Key words: causality; maturity; processes; structural equation modeling.

\section{Introdução}

A velocidade dos desdobramentos multidimensionais (social, econômico, cultural, político, ambiental, etc.) vem se acelerando a cada ano. As organizações, inseridas nesse contexto, precisam adaptar suas realidades às mudanças para garantir sua sobrevivência.

A permanente pressão, tanto no ambiente externo (tais como competição, cargas tributárias elevadas, mudanças econômicas e conjunturais) quanto interno (como a realidade da limitação de recursos e consecução de estratégias), levam a exigir respostas rápidas dos gestores de empresas [1]. Além disso, uma organização que deseja melhorias significantes necessita de uma profunda compreensão de onde ela está, isto é, qual o seu atual nível de desenvolvimento e talvez mais importante, onde ela precisa crescer (ou seja, o que deve ser melhorado para desenvolver-se ainda mais) $[2]$. 
Nesse sentido, o diagnóstico de "onde" as empresas estão pode ser fornecido pelos modelos de maturidade. Modelos de maturidade, de forma geral, partem da premissa de que as empresas se desenvolvem em estágios ou níveis de evolução, de modo que tais estágios podem ser explicitamente definidos, gerenciados e controlados ao longo do tempo [3].

Obviamente, o desenvolvimento de uma empresa perpassa a esfera de suas atividades de produção (lucrativas ou não). Todo trabalho importante realizado nas empresas faz parte de algum processo [4]. Um processo organizacional é a composição de atividades que visam à criação de valores para os clientes da empresa. Tais atividades são operadas por atores que podem ser seres humanos ou máquinas e, muitas vezes, envolvem várias unidades organizacionais que são responsáveis por todo o processo [5].

Um estudo realizado pelo IBGE (2012) [6] relata que o fluxo de entrada e saída de empresas no mercado brasileiro nos anos de 2008, 2009 e 2010 foi bem elevado. As taxas de sobrevivência para esse período (anual) estão em torno de $78 \%$, que é algo relativamente alto. Salienta-se, entretanto, que o período mais conturbado para uma nova organização são os primeiros dois anos [7] e, de acordo com o mesmo estudo, de um total de 464,7 mil empresas que apareceram pela primeira vez no mercado em 2007, 76, 1\% sobreviveram em 2008, 61,3\% sobreviveram até 2009 e pouco mais da metade $(51,8 \%)$ sobreviveram até 2010 , isto é, após três anos da entrada no mercado, quase metade $(48,2 \%)$ das empresas não sobreviveu.

Diante dessas considerações, este trabalho buscou compreender e estabelecer, por meio da modelagem de equações estruturais, a força e o sentido da relação entre construtos associados em níveis de efetividade de processos organizacionais e construtos relativos à maturidade em empresas, identificando, caso existam, os processos/construtos que melhor predizem o fator maturidade organizacional.

$\mathrm{Na}$ literatura, existem alguns trabalhos que buscaram compreender, através de técnicas de correlação canônica, a relação entre processos organizacionais e maturidade nas empresas, como é o caso de Becegato [8], Ferreira [9] e Yuki [1].

Existem também outros trabalhos que procuraram determinar a correlação entre níveis de maturidade organizacional e conjuntos de variáveis relacionadas a ou- 
tros conceitos isto é, a construtos relativos a outras áreas de conhecimento. Desses trabalhos, pode-se citar Silveira [3], que investigou a força e a direção dos construtos relacionados à MGP, Maturidade em Gestão de Pessoas, e a PTDE, Práticas de Treinamento, Desenvolvimento e Educação, de organizações. Mais recentemente, Lammers [10] investigou como ocorre e em que medida a maturidade dos processos afetam o desempenho dos negócios. Ambos os autores utilizaram a técnica de modelagem de equações estruturais.

A ideia central consiste em considerar os conceitos de processos e maturidade organizacional como construtos teóricos. Um construto é um conceito que o pesquisador pode definir em termos teóricos, mas que não pode ser diretamente medido, por exemplo, o respondente não pode articular uma única resposta que fornecerá total e perfeitamente uma medida do conceito ou medido sem erro [11]. Não importa qual o seu nível de especificidade, um construto não pode ser medido direta e perfeitamente, mas deve ser aproximadamente medido por indicadores.

Este trabalho será apresentado em 4 (quatro) seções, além da presente introdução. $\mathrm{Na}$ seção 2, a seguir, o referencial teórico concernente às técnicas estatísticas multivariadas utilizadas (análise fatorial exploratória e modelagem de equações estruturais). Na seção 3, serão expostos os procedimentos metodológicos que nortearam esta pesquisa. Finalmente, as duas seções seguintes versarão, respectivamente, sobre os resultados e algumas discussões seguidos das considerações finais.

\section{Análise multivariada}

A análise multivariada é um conjunto de métodos estatísticos utilizados em situações nas quais várias variáveis são medidas simultaneamente em cada elemento amostral $[12,13]$.

Nas seções 2.1 e 2.2, serão apresentados dois métodos da estatística multivariada: a análise fatorial exploratória e a família de técnicas de modelagem de equações estruturais, respectivamente. 


\subsection{Análise fatorial exploratória}

A análise fatorial exploratória, ou EFA (Exploratory Factorial Analysis), é uma técnica de modelagem causal que tenta "explicar"as correlações entre um conjunto de variáveis observáveis através da combinação linear de um número desconhecido de alguns fatores aleatórios latentes (variáveis não observáveis) [14].

\subsubsection{Modelo fatorial ortogonal}

Seja $X$ um vetor aleatório $p$-dimensional com vetor de médias $\mu$ e matriz de covariância $\Sigma$. No modelo fatorial, $X$ torna-se linearmente dependente sobre as variáveis aleatórias não observáveis $F_{1}, F_{2}, \ldots, F_{m}$ (com $m \leq p$ ) chamados fatores comuns e $p$ fontes de variações aditivas $\varepsilon_{1}, \varepsilon_{2}, \ldots, \varepsilon_{p}$, chamadas de erros ou fatores específicos [12]. Então, o modelo fatorial é:

$$
\begin{array}{ccc}
X_{1}-\mu_{1}= & \ell_{11} F_{1}+\ell_{12} F_{2}+\ldots+\ell_{1 m} F_{m}+\varepsilon_{1} \\
X_{2}-\mu_{2}= & \ell_{21} F_{1}+\ell_{22} F_{2}+\ldots+\ell_{2 m} F_{m}+\varepsilon_{2} \\
\vdots & \vdots & \vdots \\
X_{p}-\mu_{p}= & \ell_{p 1} F_{1}+\ell_{p 2} F_{2}+\ldots+\ell_{p m} F_{m}+\varepsilon_{p}
\end{array}
$$

ou em notação matricial:

$$
\underbrace{X-\mu}_{(p \times 1)}=\underbrace{L}_{(p \times m)} \underbrace{F}_{(m \times 1)}+\underbrace{\varepsilon}_{(p \times 1)}
$$

onde $\ell_{i j}$ é denominado como o peso ou carregamento da $i$-ésima variável no $j$ ésimo fator, de modo que a matriz $L$ é chamada matriz de cargas fatoriais.

Cumpridas certas hipóteses sobre o modelo fatorial ortogonal, sua estrutura de covariância se torna [12-14]:

$$
\Sigma=L L^{\prime}+\Psi
$$

A parte da variância da $i$-ésima variável explicada pelos $m$ fatores comuns é 
chamada de comunalidade e é dada por [12-14]:

$$
h_{i}^{2}=\ell_{i 1}^{2}+\ldots+\ell_{i m}^{2}
$$

e $\psi_{i}=\sigma_{i}^{2}-h_{i}^{2}$ são as variâncias específicas.

Existem vários métodos para se estimar os parâmetros envolvidos no modelo fatorial da equação (2.2), como o método de máxima verossimilhança (MMV), assentado na hipótese de normalidade multivariada dos dados, e o método das componentes principais (MCP), que não pressupõe a hipótese de normalidade [12-14].

A próxima seção apresenta alguns conceitos acerca da modelagem de equações estruturais.

\subsection{Modelagem de equações estruturais}

A modelagem de equações estruturais ou SEM (Structural Equation Models) não designa uma técnica estatística única, mas em vez disso refere-se a uma família de procedimentos relacionados $[11,15]$. Constitui um conjunto de equações lineares, com coeficientes a estimar, para especificar fenômenos de um determinado modelo estocástico em termos de suas presumidas relações de causa e efeito [16].

SEM é particularmente útil quando uma variável latente pode servir como causa de outra, daí o uso de equações de regressão embutidas no modelo [16].

Modelos de equações estruturais são divididos em duas partes: modelo estrutural e modelo de mensuração, conforme se verá a seguir.

\subsubsection{Modelo matemático}

O modelo estrutural representa as relações causais entre as variáveis latentes e é expresso por um conjunto de equações estruturais lineares semelhantes às equações de regressão. Matematicamente, o modelo estrutural para variáveis latentes é [13, 14]:

$$
\underbrace{\eta}_{m \times 1}=\underbrace{B}_{m \times m} \underbrace{\eta}_{m \times 1}+\underbrace{\Gamma}_{m \times n} \underbrace{\xi}_{n \times 1}+\underbrace{\zeta}_{m \times 1}
$$

em que: 
- $\eta_{m \times 1}=\left(\eta_{1}, \eta_{2}, \cdots, \eta_{m}\right)$ é o vetor aleatório de variáveis latentes endógenas;

- $\xi_{n \times 1}=\left(\xi_{1}, \xi_{2}, \cdots, \xi_{n}\right)$ é o vetor aleatório de variáveis latentes exógenas;

- $\zeta_{m \times 1}=\left(\zeta_{1}, \zeta_{2}, \cdots, \zeta_{m}\right)$ é o vetor aleatório de erros latentes;

- $\boldsymbol{B}_{m \times m}$ é uma matriz com zeros na diagonal e representa as relações de construtos endógenos $\eta$ com outros construtos endógenos $\eta$;

- $\Gamma_{m \times n}$ representa as relações de construtos exógenos $\xi$ com endógenos $\eta$.

Já o modelo de mensuração que relaciona as variáveis não observáveis às variáveis observáveis é:

$$
\begin{aligned}
& \underbrace{Y}_{p \times 1}=\underbrace{\Lambda_{y}}_{p \times m} \underbrace{\eta}_{m \times 1}+\underbrace{\varepsilon}_{p \times 1} \\
& \underbrace{X}_{q \times 1}=\underbrace{\Lambda_{x}}_{q \times n} \underbrace{\xi}_{n \times 1}+\underbrace{\delta}_{q \times 1}
\end{aligned}
$$

em que:

- $Y_{p \times 1}=\left(y_{1}, y_{2}, \cdots, y_{p}\right)$ é o vetor aleatório de indicadores observados do vetor latente endógeno $\eta_{m \times 1}$;

- $X_{q \times 1}=\left(x_{1}, x_{2}, \cdots, x_{q}\right)$ é o vetor aleatório de indicadores observados do vetor latente exógeno $\xi_{n \times 1}$;

- $\varepsilon_{p \times 1}=\left(\varepsilon_{1}, \varepsilon_{2}, \cdots, \varepsilon_{p}\right)$ e $\boldsymbol{\delta}_{q \times 1}=\left(\delta_{1}, \delta_{2}, \cdots, \delta_{q}\right)$ são vetores aleatórios representando os erros de mensuração de $Y_{p \times 1}$ e $X_{q \times 1}$, respectivamente;

- $\Lambda_{y_{(p \times m)}}$ e $\Lambda_{x_{(q \times n)}}$ são as matrizes dos coeficientes de regressão relacionando $Y_{p \times 1} \operatorname{com} \eta_{m \times 1}$, e $X_{q \times 1} \operatorname{com} \xi_{n \times 1}$, respectivamente.

Logo, o modelo de equações estruturais conjunto é:

$$
\begin{aligned}
& \eta=B \eta+\Gamma \xi+\zeta \\
& Y=\Lambda_{y} \eta+\varepsilon \\
& X=\Lambda_{x} \xi+\delta
\end{aligned}
$$


Assim, verificadas certas hipóteses sobre o modelo dado em (2.7), a matriz de covariância do vetor aleatório de variáveis indicadoras $Z_{\{(p+q) \times 1\}}^{\prime}=\left[Y_{p \times 1}^{\prime} \vdots X_{q \times 1}^{\prime}\right]$, se torna dependente do vetor de parâmetros desconhecidos $\theta=\left(\boldsymbol{B}_{m \times m}, \boldsymbol{\Gamma}_{m \times n}\right.$, $\left.\Lambda_{y_{p \times m}}, \Lambda_{x_{q \times n}}, \Phi, \Psi, \Theta_{\varepsilon}, \Theta_{\delta}\right)$, isto é:

$$
\Sigma(\theta)=\left[\begin{array}{cc}
\Lambda_{y}(I-B)^{-1}\left(\Gamma \Phi \Gamma^{\prime}+\Psi\right)\left[(I-B)^{-1}\right]^{\prime} \Lambda_{y}^{\prime}+\Theta_{\varepsilon} & \Lambda_{y}(I-B)^{-1} \Gamma \Phi \Lambda_{x}^{\prime} \\
\Lambda_{x} \Phi \Gamma^{\prime}\left[(I-B)^{-1}\right]^{\prime} \Lambda_{y}^{\prime} & \Lambda_{x} \Phi \Lambda_{x}^{\prime}+\Theta_{\delta}
\end{array}\right]
$$

em que $\Phi, \Psi, \Theta_{\varepsilon}$ e $\boldsymbol{\Theta}_{\delta}$ são as matrizes de covariâncias de $\xi_{n \times 1}, \zeta_{m \times 1}, \varepsilon_{p \times 1}$ e $\boldsymbol{\delta}_{q \times 1}$, respectivamente.

Tabela 1. Fórmulas e descrição dos diversos indices de ajuste

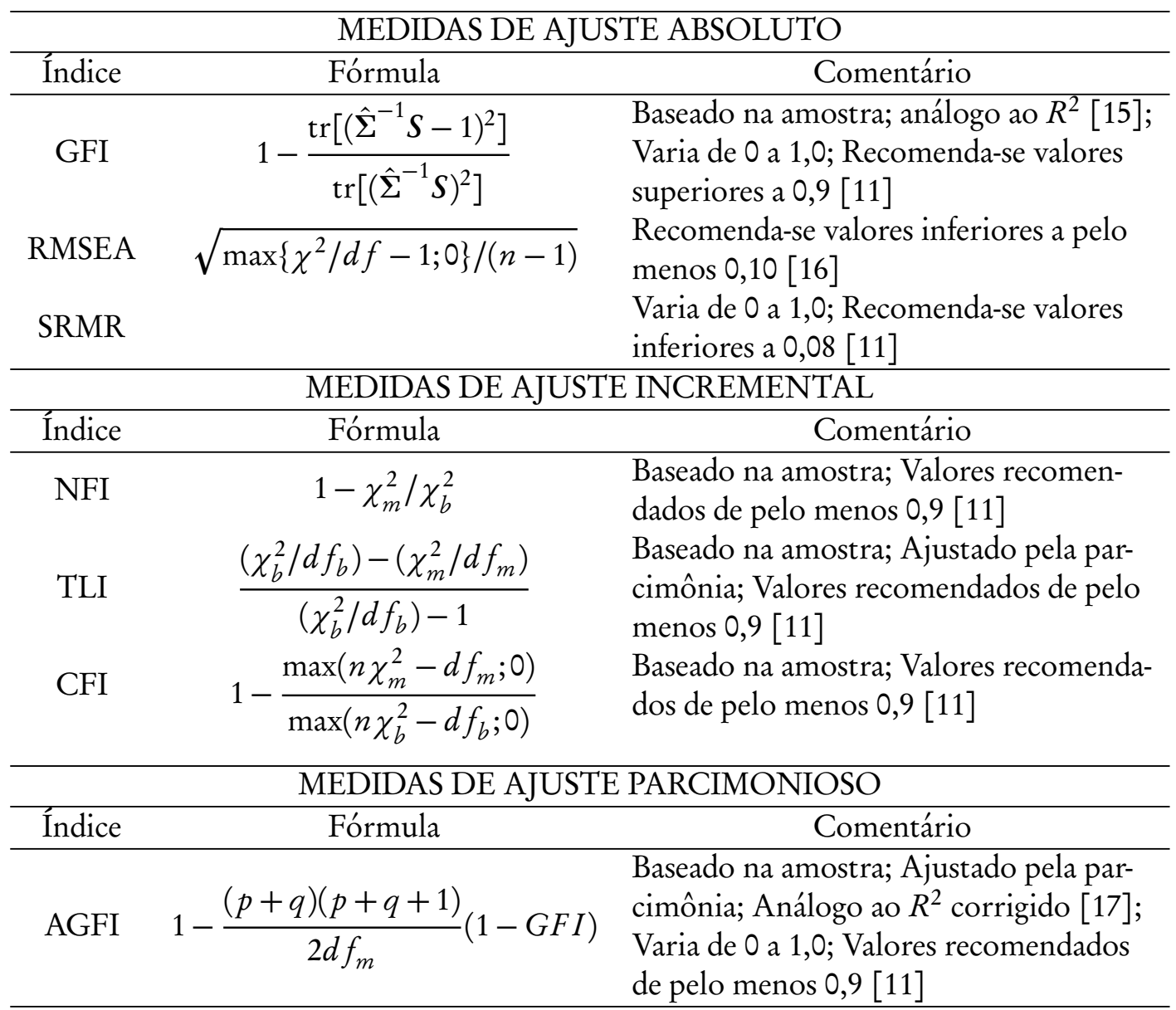

Fonte: Adaptado de Hair et al. [11]; Kline [15]; Loesch e Hoeltgebaum [16] 
Uma das vantagens das técnicas de modelagem de equações estruturais é que existe um grande número de índices que indicam se o modelo proposto se ajustou bem aos dados coletados. A qualidade de ajuste a que se referem esses índices, mede a correspondência da matriz de dados de entrada reais ou observados (covariâncias ou correlações) com aquela prevista com o modelo proposto [11].

$\mathrm{Na}$ tabela 1, encontra-se a descrição dos principais índices de ajuste com os respectivos valores de referência (comumente aceitos pela literatura).

Para estimar os parâmetros de $\Sigma(\theta)$ em (2.8), obtém-se uma estimativa amostral $S$ de $\Sigma(\theta)$ e escolhe-se uma função de ajuste $F(S, \Sigma(\theta)) \geq 0$, contínua, que é mini-

mizada em $\theta=\hat{\theta}$, de modo que o valor da função em $\Sigma(\hat{\theta})=\hat{\Sigma}$, representado como $F(S, \hat{\Sigma})$, é uma medida de proximidade do ajuste de $S$ por $\hat{\Sigma}$ [14].

Duas funções de ajuste comuns para SEM são as funções de máxima verossimilhança, $F_{M L}$ (Maximum Likelihood) e de mínimos quadrados generalizados, $F_{G L S}$ (Generalized Least Squares), descritas na equação (2.9) [14]:

$$
\begin{aligned}
& F_{M L}(S, \Sigma(\theta))=\ln |\Sigma(\theta)|+\operatorname{tr}\left(S \Sigma(\theta)^{-1}\right)-\ln (S)-(p+q) \\
& F_{G L S}(S, \Sigma(\theta))=\frac{1}{2} \operatorname{tr}\left[S^{-1}(S-\Sigma(\theta))\right]^{2}=\frac{1}{2}\left\|S^{-1}(S-\Sigma(\theta))\right\|^{2}
\end{aligned}
$$

Na próxima seção, serão apresentados a metodologia e os procedimentos adotados nas análises.

\section{Desenvolvimento}

O banco de dados utilizado neste trabalho consiste de uma planilha composta por 299 observações (empresas dos mais variados segmentos, sendo, a maioria, de Curitiba e região metropolitana) por 42 variáveis, coletadas no período de 2008.

É de grande interesse verificar se os dados a serem manuseados suportam a hipótese de normalidade, pois, conforme equação (2.9), algumas técnicas dependem dessa condição. Para isso, utilizou-se o teste de normalidade multivariada proposto por Doornik-Hansen [18]. Esse teste apresentou um $p$-valor aproximadamente nulo, indicando que a normalidade deve ser rejeitada. Por essa razão, as estimativas necessárias nas análises subsequentes foram realizadas com métodos que indepen- 
dem da hipótese de normalidade.

\subsection{Maturidade}

Não se conhecia a priori a estrutura fatorial do conjunto de variáveis relativas ao conceito de maturidade organizacional, razão pela qual esse conjunto foi investigado segundo a análise fatorial exploratória.

Antes de aplicar essa técnica, realizou-se um teste de hipótese (chamado teste de esfericidade de Bartlett), a fim de analisar se as variáveis eram correlacionadas e calculou-se uma medida (denominada medida de adequação da amostra) que pudesse exprimir a adequação dessas correlações, com o objetivo de verificar se o tratamento fatorial seria viável.

O teste de esfericidade de Bartlett tem como hipótese inicial que a matriz de correlação é igual à matriz identidade, ou seja, as correlações não são significativas. $\mathrm{O}$ $p$-valor encontrado nesse teste foi aproximadamente nulo, indicando que a matriz de correlações possui correlações significativas. A medida de adequação da amostra, desenvolvida por Kaiser-Meier-Olkin, apresentou valor $K M O=0,883$ significando que o ajuste fatorial é bom $[13,19]$.

As estimativas para a análise fatorial exploratória foram obtidas por meio de uma função implementada no software Matlab 7.9.0, e tais resultados apresentam-se na tabela 2. As cargas e as comunalidades $h^{2}$ (tabela 2) foram estimadas pelo método das componentes principais (MCP).

Observa-se da tabela 2 que o modelo fatorial explica 64,58\% da variância das variáveis observadas. Os fatores foram interpretados como:

- Fator Mercado (F1) - X1: Qual o nível de investimentos em tecnologias e equipamentos realizados nos últimos 3 anos? X2: Qual a posição da empresa em relação às concorrentes? X3: Qual o tempo de resposta a demandas de mercado? (novos produtos, certificações, novas tecnologias) X4: Qual é a capacidade da empresa em se adaptar às mudanças do seu mercado / ambiente? X5: Com relação a sua empresa você diria [ela segue as mudanças no mercado]? 
Tabela 2. Cargas e comunalidades $\left(b^{2}\right)$ estimadas pelo método das componentes principais (MCP)

\begin{tabular}{c|ccccc}
\hline \multirow{2}{*}{ VARIÁVEL } & \multicolumn{4}{|c}{ FATORES/COMUNALIDADES } \\
\cline { 2 - 5 } & F1 & F2 & F3 & $b^{2}$ \\
\hline X1 & $-0,163$ & 0,149 & 0,825 & 0,729 \\
X2 & $-0,284$ & 0,107 & 0,751 & 0,655 \\
X3 & $-0,139$ & 0,548 & 0,421 & 0,496 \\
X4 & $-0,160$ & 0,711 & 0,257 & 0,596 \\
X5 & $-0,243$ & 0,792 & 0,056 & 0,690 \\
X6 & $-0,134$ & 0,790 & 0,113 & 0,656 \\
X7 & $-0,536$ & 0,275 & 0,538 & 0,652 \\
X8 & $-0,678$ & 0,193 & 0,197 & 0,536 \\
X9 & $-0,788$ & $-0,062$ & 0,177 & 0,656 \\
X10 & $-0,847$ & 0,190 & 0,139 & 0,772 \\
X11 & $-0,777$ & 0,314 & 0,212 & 0,747 \\
X12 & $-0,616$ & 0,347 & 0,252 & 0,564 \\
\hline Proporcẫo Explicada (\%) & 44,54 & 11,91 & 8,14 \\
Proporção Acumulada (\%) & 44,54 & 56,44 & 64,58 \\
\hline \multicolumn{5}{|c}{} \\
\hline
\end{tabular}

- Fator Funcionários (F2) - X6: Qual o grau de autonomia dos funcionários? X7: Como você classifica o estilo de gestão da empresa? X8: Qual o nível de cooperação entre os funcionários? X9: Qual o nível de interação (troca de informações formais e informais) entre os funcionários?

- Fator Formalização (F3) - X10: Qual a quantidade de horas de treinamento dos funcionários por ano? X11: Qual o nível de formalização das atividades e processos? X12: Existe uma estratégia organizacional claramente definida?

A partir desses resultados, especificou-se um modelo fatorial hierárquico, isto é, de segunda ordem, conforme diagrama de caminhos na figura 1. Esse construto de segunda ordem, denominado fator Maturidade (Mat), explica 70,44\% da variância oriunda dos três construtos de primeira ordem (fator Mercado: F1; fator Funcionários: F2; e fator Formalização: F3) e será útil na especificação do modelo estrutural que considera as relações de causalidade entre maturidade e processos, o qual será tratado na seção 3.3, a seguir.

Os coeficientes de caminho (inseridos na figura 1), bem como as medidas de qualidade de ajuste (tabela 3) para esse modelo foram estimadas pelo método de mínimos quadrados generalizados (GLS) (que não supõe normalidade multivariada 
Revista Ciências Exatas e Naturais, Vol.14, n², Jul/Dez 2012

$[16,17])$, através do pacote sem, do software $R$, versão 2.15.0.

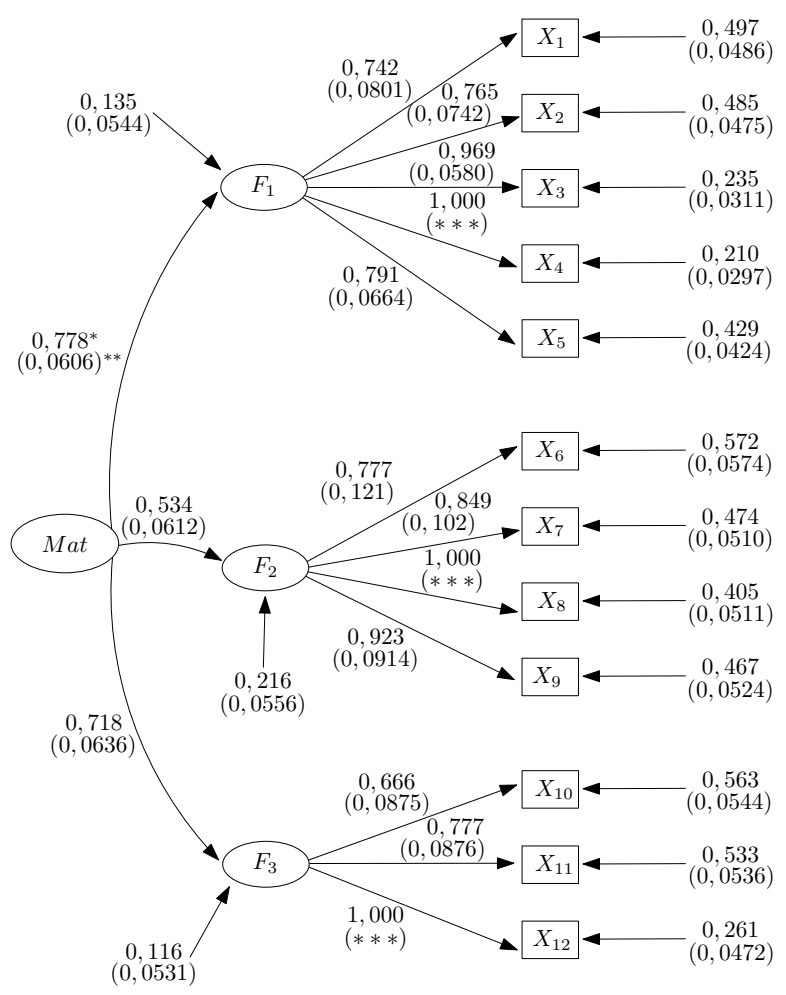

Figura 1. Diagrama de caminhos para modelo hierárquico de maturidade

Nota: * Cargas estimadas, ${ }^{* *}$ erros padrões entre parênteses $\mathrm{e}^{* * *}$ cargas fixadas em 1,000 para padronização dos fatores

As medidas de qualidade de ajuste (tabela 3 ) indicam que o modelo foi bem ajustado (conforme valores enunciados na tabela 1 ).

Tabela 3. Medidas de qualidade de ajuste para o modelo fatorial confirmatório de segunda ordem diagramado pela figura 1

\begin{tabular}{c|c|c|c|c|c|c|c|c|c}
\hline MÉTODO & $\chi^{2}$ & $d f$ & GFI & AGFI & RMSEA & NFI & TLI & CFI & SRMR \\
\hline GLS & 121,01 & 51 & 0,932 & 0,897 & 0,0679 & 0,968 & 0,976 & 0,981 & 0,0754 \\
\hline
\end{tabular}

Como se está trabalhando com fatores latentes, faz-se necessário avaliar as confiabilidades dos construtos considerados. Confiabilidade trata-se da extensão em que uma variável ou conjunto de variáveis é consistente com o que se pretende medir [11], isto é, confiabilidade é o grau de ausência de erro sistemático em uma medida. 
Os fatores de primeira ordem apresentaram boas confiabilidades [11, 15], ambas medidas por meio do coeficiente alfa de Cronbach, cujas estimativas foram $\alpha_{F 1}=0,8546, \alpha_{F 2}=0,7620$ e $\alpha_{F 3}=0,7433$. Já a confiabilidade do fator Maturidade (Mat) de segunda ordem foi estimada por meio do coeficiente de confi-

abilidade composta $\rho_{\text {Mat }}^{2}=0,8747$ (que envolve as estimativas da análise fatorial confirmatória [17]), indicando também uma boa confiabilidade.

\subsection{Processos}

Para o conjunto de variáveis relativas a processos empresariais, conhecia-se o agrupamento das variáveis, uma vez que a elaboração dessas variáveis deu-se com base no modelo Process Classification Framework (PCF) [20]. O PCF é uma arquitetura utilizada para organizar e divulgar o conhecimento das melhores práticas, isto é, das melhores formas que as organizações realizam determinadas atividades [21]. Assim, os construtos considerados são formados da seguinte maneira:

- Fator Desenvolvimento de Visão e Estratégica (F1P) - X13: Sua empresa avalia o impacto das mudanças sociais e culturais na sua estratégia? X14: A empresa possui uma visão estratégia alinhada ao negócio? X15: Empresa procura sempre difundir essa visão estratégica? X16: A empresa busca sempre administrar as metas para então, medi-las e avaliá-las?

- Fator Desenvolver e Gerenciar Produtos e Serviços (F2P) - X17: A empresa utiliza estratégias na concepção e no desenvolvimento de novos produtos? X18: A escolha e avaliação de novos produtos levam em consideração as ideias e necessidades dos consumidores? X19: O projeto, fabricação e evolução dos produtos estão relacionados com a qualidade e confiabilidade? X20: Os testes ou avaliação dos produtos são realizados através de apresentação aos clientes e entrevistas? X21: Antes do lançamento de um novo produto são preparados protótipos e realizados testes?

- Fator Marketing de Produtos e Serviços (F3P) - X22: A empresa possui marketing direcionado para os seus produtos e serviços? X23: A empresa conhece 
seu mercado de atuação através da sua segmentação? X24: A empresa possui estratégias de vendas, previsão de vendas e administra essas estratégias? X25: A empresa possui orçamentos de vendas globais, estabelece métricas para esses orçamentos e efetua o seu controle?

- Fator Entrega de Produtos e Serviços (F4P) - X26: Existe na empresa um planejamento da cadeia de suprimentos, em que a empresa administra as demanda dos produtos e serviços? X27: A empresa identifica as matérias prima críticas? X28: Há um controle de tempo e metas nos processos de produção? X29: A empresa faz previsão de demanda? X30: A empresa possui controle de estoque através de inventários? X31: A empresa tem uma programação baseada em planos de produção? X32: A empresa possui um layout (arranjo físico) definido / apropriado?

- Fator Gerência do Atendimento ao Consumidor (F5P) - X33: A empresa administra os contratos com os clientes correlacionando-os com as requisições e ordens de produção ou serviço? X34: A empresa possui uma logística que traduz as exigências e o bom atendimento ao consumidor? X35: A empresa possui políticas em relação ao atendimento ao consumidor e as gere através de medidas de satisfação do cliente? X36: A empresa analisa através de dados a satisfação dos serviços e dos produtos que coloca no mercado com o intuito de identificar melhorias?

- Fator Gerenciamento de Recursos Financeiros (F6P) - X37: A empresa utiliza a contabilidade como fonte de informação para o planejamento e controle? X38: A empresa possui políticas de orçamento? X39: A empresa possui controles analíticos e sintéticos das receitas e despesas e através dessas efetua a análise de discrepância de acordo com orçamentos pré-estabelecidos? X40: A empresa possui estratégia definida para a gestão dos tributos federais, estaduais e municipais? X41: A empresa possui procedimentos de auditorias externas de forma periódica? X42: A empresa controla seus lucros contábeis e financeiros e administra o risco do negócio pautado em dados confiáveis e controláveis? 
Estes seis construtos apresentaram confiabilidades por consistência interna em torno de 0,9 $\left(\alpha_{F 1 P}=0,899 ; \alpha_{F 2 P}=0,912 ; \alpha_{F 3 P}=0,888 ; \alpha_{F 4 P}=0,933 ; \alpha_{F 5 P}=\right.$ 0,892 e $\alpha_{F 6 P}=0,930$ ), o que indica ótimas confiabilidades [11].

\subsection{Modelo de equação estrutural}

Após a avaliação da confiabilidade dos construtos considerados, especificou-se um modelo de equação estrutural entre os seis construtos/processos e o fator de segunda ordem Maturidade (Mat), apresentados nas seções 3.1 e 3.2, respectivamente.

A especificação seguiu o padrão apresentado no diagrama de caminhos da figura 2. Nesse diagrama, observa-se que o construto de segunda ordem Mat (Maturidade) é suposto ser causado pelo fator F1P (Desenvolvimento de Visão e Estratégia), através da relação $\mathrm{F} 1 \mathrm{P} \longrightarrow$ Mat e o coeficiente de caminho dessa relação indica a força de causalidade procurada. Outro detalhe a salientar é que os coeficientes de caminho, bem como os erros padrões (números apresentados entre parênteses), estão inseridos nesse diagrama (figura 2). A especificação para os construtos/processos restantes podem ser diagramadas de maneira equivalente.

A tabela 4 apresenta os índices de qualidade de ajuste bem como as estimativas dos coeficientes de caminho. Analisando os índices de qualidade de ajuste, observase que todos eles indicam adequação do modelo especificado, pois estão dentro dos limites estabelecidos na literatura (compare valores com a tabela 1).

Tabela 4. Medidas de qualidade de ajuste para os modelos de equações estruturais individuais conforme figura 2

\begin{tabular}{cccccccccc}
\hline MODELO & $\chi^{2}$ & $d f$ & GFI & AGFI & RMSEA & NFI & TLI & CFI & SRMR \\
\hline F1P $\rightarrow$ MAT & 236,73 & 100 & 0,901 & 0,865 & 0,0677 & 0,975 & 0,982 & 0,985 & 0,07833 \\
F2P $\rightarrow$ MAT & 239,98 & 115 & 0,905 & 0,874 & 0,0604 & 0,977 & 0,985 & 0,988 & 0,07556 \\
F3P $\rightarrow$ MAT & 184,16 & 100 & 0,923 & 0,895 & 0,0531 & 0,98 & 0,989 & 0,991 & 0,07672 \\
F4P $\rightarrow$ MAT & 276,96 & 148 & 0,902 & 0,874 & 0,0541 & 0,979 & 0,988 & 0,990 & 0,09584 \\
F5P $\rightarrow$ MAT & 204,93 & 100 & 0,914 & 0,883 & 0,0593 & 0,975 & 0,985 & 0,987 & 0,07779 \\
F6P $\rightarrow$ MAT & 243,51 & 131 & 0,909 & 0,882 & 0,0537 & 0,982 & 0,990 & 0,991 & 0,08949 \\
\hline
\end{tabular}

Dos seis processos analisados, aquele que tem maior impacto nos níveis de maturidade é o primeiro (ver tabela 5), isto é, o construto Desenvolvimento de Visão e Estratégia que possui o maior coeficiente de caminho $\mathrm{F} 1 \mathrm{P} \rightarrow$ Mat $=0,727$, seguido 
Revista Ciências Exatas e Naturais, Vol.14, n², Jul/Dez 2012

do fator Gerência do Atendimento ao Consumidor $(\mathrm{F} 5 \mathrm{P} \rightarrow$ Mat $=0,607)$ e fator Marketing de Produtos e Serviços (F3P $\rightarrow$ Mat =0,607). De um modo geral todas as relações de causalidade podem ser consideradas como efeitos “grandes”, já que são $>0,50[15]$.

Tabela 5. Coeficientes de caminho estimados por Mínimos Quadrados Generalizados (GLS)

\begin{tabular}{cccccc}
\hline MODELO & CARGA $^{*}$ & $p$-VALOR & $R^{2}$ & VAR. ESPEC. & $p$-VALOR \\
\hline F1P $\rightarrow$ MAT & $0,727(0,0582)^{* *}$ & 0,000 & 0,873 & $0,077(0,0250)$ & 0,002 \\
F2P $\rightarrow$ MAT & $0,541(0,0684)$ & 0,000 & 0,628 & $0,174(0,0342)$ & 0,000 \\
F3P $\rightarrow$ MAT & $0,607(0,0600)$ & 0,000 & 0,699 & $0,159(0,0336)$ & 0,000 \\
F4P $\rightarrow$ MAT & $0,554(0,0670)$ & 0,000 & 0,655 & $0,162(0,0353)$ & 0,000 \\
F5P $\rightarrow$ MAT & $0,607(0,0598)$ & 0,000 & 0,707 & $0,153(0,0321)$ & 0,000 \\
F6P $\rightarrow$ MAT & $0,560(0,0659)$ & 0,000 & 0,630 & $0,184(0,0354)$ & 0,000 \\
\hline
\end{tabular}

Nota: * Carga padronizada. ${ }^{* *}$ Erros padrões entre parênteses

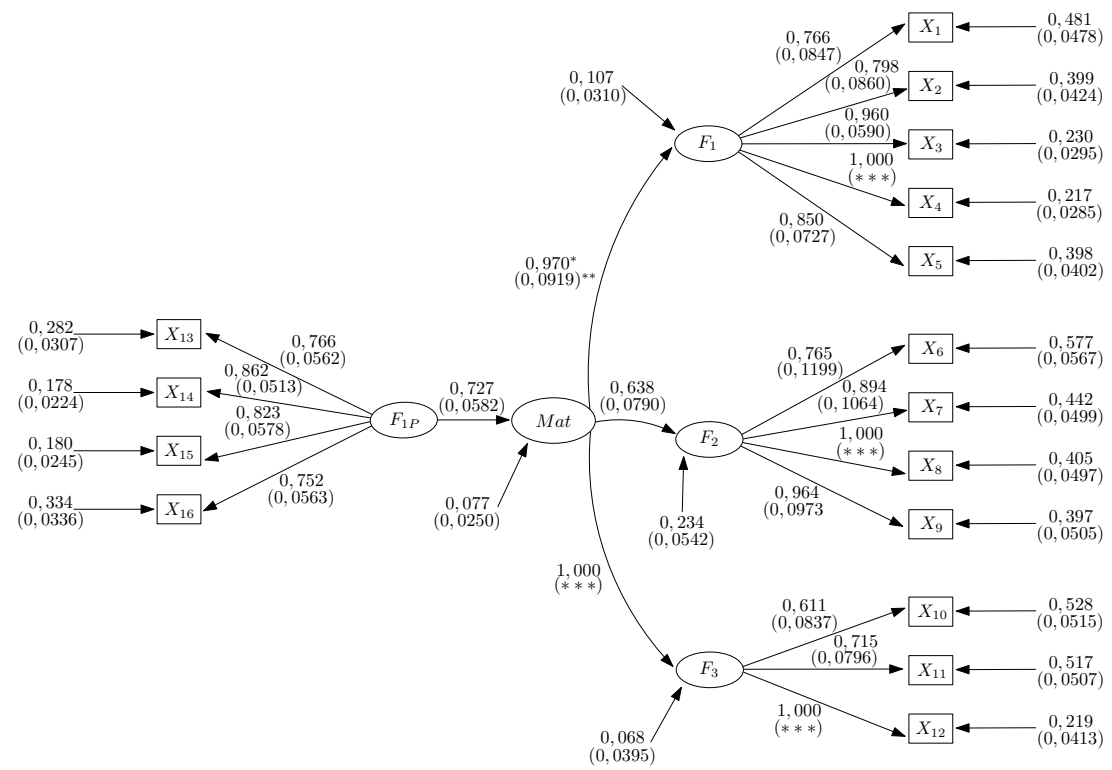

Figura 2. Diagrama de caminhos para o modelo estrutural que estabelece o construto F1P como causa de Mat

Nota: * Cargas estimadas, ${ }^{* *}$ erros padrões entre parênteses $\mathrm{e}^{* * *}$ cargas fixadas em 1,000 para padronização dos fatores

O fator mais importante é o construto Desenvolvimento de Visão e Estratégia 
$\left(P_{1 P}\right)$. Observa-se que $87,3 \%\left(R^{2}=0,873\right)$ da variância do fator de ordem superior Maturidade (Mat) é explicada pelo construto Desenvolvimento de Visão e Estratégia. Conforme tabela 5, este é o maior $R^{2}$. O segundo construto mais importante, conforme a ordem de grandeza dos coeficientes de caminho e de $R^{2}$, é o construto Gerência do Atendimento ao Consumidor (F5P). Esse construto explica 70,7\% da variância do fator Maturidade $\left(R^{2}=0,707\right)$. O construto Marketing de Produtos e Serviços (F3P) se equipara em grau de importância (carga e $R^{2}$ ) ao construto F5P.

Os demais construtos possuem pesos, bem como coeficientes de determinação $R^{2}$, em torno de 0,55 que também são de ordens significativas [15].

\section{Conclusões}

A partir dos modelos estruturais hipotetizados que buscaram analisar relações de causalidade entre processos organizacionais e maturidade das empresas, pode-se verificar que todas as relações estipuladas são fortes. É possível, portanto, concluir que os processos estudados são bons preditores para o conceito latente maturidade organizacional e níveis superiores de desenvolvimento de processos resultam níveis superiores de maturidade. Ou, dito de outra forma, o desenvolvimento dos processos nas organizações ajudam a explicar o seu próprio nível de desenvolvimento (maturidade).

Os processos mais importantes, dentre aqueles analisados, são os processos Desenvolvimento de Visão e Estratégia, Gerência do Atendimento ao Consumidor e Marketing de Produtos e Serviços, que apresentaram os maiores coeficientes estruturais e de determinação $R^{2}$.

Dessa forma, apesar de não ser o caso ignorar os outros processos organizacionais analisados e, a fim de estabelecer maiores níveis de maturidade, a ênfase deve ser dada à manutenção das relações associadas ao desenvolvimento de visão e estratégia da organização (F1P) e na gerência do atendimento ao consumidor (F5P), pois a dependência do construto maturidade é mais intensa para esses dois fatores.

Modelos de maturidade são mecanismos de busca por melhoria contínua, por essa razão, espera-se que os gestores levem em consideração esses apontamentos, a fim de auxiliar na tomada de decisão no que tange à busca por excelência no mercado 
em que sua organização se encontra.

Por fim, com a finalidade de estabelecer um estudo (ainda mais) completo sobre causalidade entre níveis de desenvolvimento de processos e maturidade organizacional, recomenda-se, para estudos futuros, a construção de escalas para outros processos não considerados neste trabalho.

\section{Referências}

[1] YUKI, W. S. Análise de correlação entre os níveis de efetividade dos processos de gestão em empresas de construção civil e a maturidade organizacional. Dissertação de Mestrado, Universidade Tecnológica Federal do Paraná. Curitiba, 2012.

[2] GRANT, K. P.; PENNYPACKER, J. S. Project management maturity: an assessment of project management capabilities among and between selected industries. IEEE T Eng Manage, v. 53, n. 1, p.59-68, 2006.

[3] SILVEIRA, V. N. S. Maturidade em gestão de pessoas e práticas de treinamento, desenvolvimento e educação: uma aplicação a partir do People Capability Maturity Model (P-CMM). Dissertação de Mestrado, Universidade Federal de Minas Gerais. Belo Horizonte, 2007.

[4] GONÇALVES, J. E. L. As empresas são grandes coleções de processos. Revista de Administração de Empresas, v. 40, n. 1, p. 6-19, 2000.

[5] LIN, F. R.; YANG, M. C.; PAI, Y. H. A generic structure for business process modeling. Lect Notes Comput Sc, v. 8, n. 1, p. 19-41, 2002.

[6] IBGE Demografia das empresas 2010. Instituto Brasileiro de Geografia e Estatística, 2012.

[7] SEBRAE Coleção estudos e pesquisas: outubro/2011. Taxa de sobrevivência das empresas no Brasil. Serviço Brasileiro de Apoio às Micro e Pequenas Empresas, 2011. 
[8] BECEGATO, A. C. Análise da correlação entre maturidade e níveis de desenvolvimento dos processos. Dissertação de Mestrado, Pontifícia Universidade Católica do Paraná. Curitiba, 2009.

[9] FERREIRA, E. D. Correlação canônica entre as características organizacionais e os níveis de processos no desenvolvimento de produto. Dissertação de Mestrado, Pontifícia Universidade Católica do Paraná. Curitiba, 2009.

[10] LAMMERS, S. V. Modeling business process maturity, performance, innovativeness, size and age of organizations: an empirical investigation into manufacturing and R\&D industries, in Germany and the Netherlands. Dissertação de Mestrado, Eindhoven University of Technology (TUE) / Berlin. Berlin, 2012.

[11] HAIR, J. F.; ANDERSON, R. E.; TATHAM, R. L.; BLACK, W. C. Applied multivariate statistical analysis. Prentice Hall, New York, 2009.

[12] JOHNSON, R. A.; WICHERN, D. W. Applied multivariate statistical analysis. Prentice Hall, New Jersey, 2007.

[13] MINGOTI, S. A. Análise de dados através de métodos de estatística multivariada: Uma abordagem aplicada. Editora UFMG, Belo Horizonte, 2005.

[14] TIMM, N. H. Applied multivariate analysis. Springer, New York, 2002.

[15] KLINE, R. B. Principles and practice of structural equation modeling. The Guilford Press, New York, 2005.

[16] LOESCH, C.; HOELTGEBAUM, M. Métodos estatísticos multivariados. Saraiva, São Paulo, 2012.

[17] LATTIN, J. M.; CARROL, J. D.; GREEN, P. E. Análise de dados multivariados. Cengage Learning, São Paulo, 2011.

[18] DOORNIK, J. A.; HANSEN, H. An omnibus test for univariate and multivariate normality. Oxford B Econ Stat, v. 70, p. 927-939, 2008. 
Revista Ciências Exatas e Naturais, Vol.14, n², Jul/Dez 2012

[19] FÁVERO, L. P.; BELFIORE, P; SILVA, F. L.; CHAN, B. L. Análise de dados: modelagem multivariada para tomada de decisões. Elsevier, Rio de Janeiro, 2009.

[20] APQC Process classification framework. 2012. Disponível em http://www.apqc.org/knowledge-base/download. Acesso em set/2012.

[21] O'LEARY, D. E. A comparative analysis of the evolution of a taxonomy for best practices: a case for "knowledge efficiency". Intelligent Systems in Accounting, Finance E Management, v. 16, n. 4, p. 293-309, 2009. 